\title{
comportement expérimental des roches sous contraintes et déformations triaxiales
}

\author{
E. GAZIEV* \\ Docteur es Sciences techniques
}

A. MOROZOV*

Ingénieur Département des Fondations aux rochers

V. CHAGANIAN*

Ingénieur Département des Fondations aux rochers Institut Hydroproject, Moscou

Les massifs rocheux dans les conditions naturelles se trouvent pratiquement toujours dans un état de contrainte triaxial, c'est pourquoi l'étude de la résistance et de la déformabilité des massif́s rocheux sous un chargement triaxial présente de l'intérêt pour l'élaboration des excavations souterraines et des projets des fondations rocheuses des grands ouvrages.

L'étude de la résistance et de la déformabilité des échantillons de roches en laboratoire s'effectue, habituellement, sous un chargement uniaxial pour lequel sont déterminées les caractêristiques telles que: résistance à la compression, résistance à la traction, module de déformation, coefficient de Poisson, etc.

Dans un état triaxial de contrainte et de déformation toutes ces caractéristiques deviennent fonctions des contraintes principales et de leurs relations réciproques, ce qui complique considérablement leur définition et, dans ces conditions, la notion de «résistance» demande à être précisée, car définir cette résistance comme contrainte maxima que le matériau est capable de supporter sans rupture, n'est pas complètement satisfaisant.
Premièrement, il est nécessaire de préciser la notion de rupture, car celle-ci commence presque dès le début de la sollicitation du matériau et continue pendant l'accroissement de la charge, en passant d'un niveau à l'autre; et, deuxièmement, les matériaux rocheux lorsqu'ils sont confinés sont capables de supporter des charges croissantes. L'échantillon n'ayant pas la possibilité de "se désagréger », le processus de sa rupture continue même pendant le passage du niveau microscopique au niveau macroscopique.

Ainsi, dans les conditions triaxiales de contrainte et de déformation la «résistance du matériau rocheux sera définie par une combinaison déterminée des contraintes principales qui provoque le commencement de la macrorupture et un changement des propriétés du matériau tel qu'il ne lui permet pas de satisfaire aux conditions initiales nécessaires à son utilisation. Le critère du changement des propriétés du matériau est, généralement, une brusque variation de sa déformabilité correspondant au passage, dans le processus de formation des fissures, du niveau microscopique au niveau macroscopique.

* Volokolamskoie chaussée, 125080 Moscou, URSS. 
Le système des contraintes principales $\sigma_{1}, \sigma_{2}$ et $\sigma_{3}$ qui provoque la rupture, peut étre représenté par un point dans l'espace des contraintes principales et l'ensemble de tels points peut être décrit par la surface:

$$
f\left(\sigma_{1}, \sigma_{2}, \sigma_{3}\right)=0
$$

caractérisant la résistance du matériau dans l'état de contrainte triaxial.

Nous admettrons dans le présent rapport que $\sigma_{1}$, est la contrainte principale majeure et $\sigma_{3}$ la contrainte principale mineure $\left(\sigma_{1}>\sigma_{2}>\sigma_{3}\right)$, la compression étant positive.

\section{ETUDE DE LA RÉSISTANCE}

La dilatation latérale libre du matériau rocheux n'ayant pas la possibilité de se manifester sous la charge, laugmentation de l'une des contraintes principales provoque l'augmentation des deux autres contraintes. De plus, l'augmentation des contraintes latérales a lieu aussi, une fois la limite de la résistance du matériau atteinte. Cette augmentation de la contrainte latérale assure l'accroissement de la capacité portante. L'exception concerne seulement les zones du massif rocheux adjacentes à la surface libre ou à l'excavation souterraine sans soutènements, dans lesquelles se crée un état de contrainte biaxial et non triaxial.

Ainsi, au cours de l'étude expérimentale de la résistance du matériau dans l'état de contrainte triaxial il est indispensable d'assurer non seulement l'application de la charge triaxiale (satisfaire aux conditions de contrainte), mais aussi la restriction correspondante des déformations volumétriques (satisfaire aux conditions de déformabilité), conditionnées par les caractéristiques de déformation du matériau étudié.

Dans ce but, au Laboratoire de mécanique des roches de l'Institut "Hydroproject " a été mise au point une installation spéciale permettant de tester un échantillon cubique de $20 \times 20 \times 20 \mathrm{~cm}$ de dimensions en reproduisant la rigidité du massif encaissant.

L'impossibilité d'avoir une dilatation latérale libre de l'échantillon conduit à une augmentation, par rapport à leurs valeurs initiales, des contraintes $\sigma_{2}$ et $\sigma_{3}$ proportionnellement à l'augmentation de la contrainte $\sigma_{1}$.

Après la rupture de l'échantillon quí est définie par un brusque changement des diagrammes de déformations et par une brusque augmentation de l'intensité de l'émission acoustique, l'accroissement des contraintes $\sigma_{2}$ et $\sigma_{3}$ continue avec l'accroissement de $\sigma_{1}$, mais avec un autre facteur de proportionnalité.

Il en résulte qu'une fois l'échantillon sur la surface de résistance $\mathrm{f}\left(\sigma_{1}, \sigma_{2}, \sigma_{3}\right)=0$, il ne la quitte plus et, avec l'augmentation de la contrainte $\sigma_{1}$, "glisse" le long de la surface.

Sur la figure la est représentée l'enveloppe des cercles de Mohr construits d'après les résultats d'essais des échantillons dans l'état de contrainte triaxial à $\sigma_{2}=\sigma_{3}$, et sur la figure 16 sont montrés les cercles de Mohr

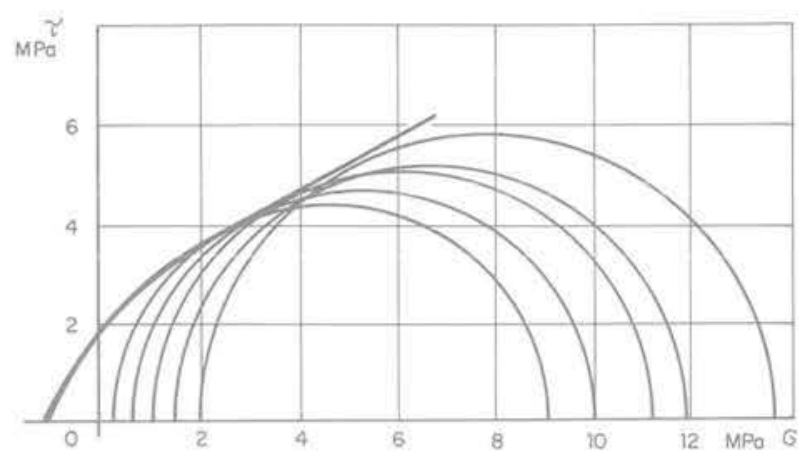

Fig. 1a. - Courbe enveloppe de Mohr pour différents résultats expérimentaux.

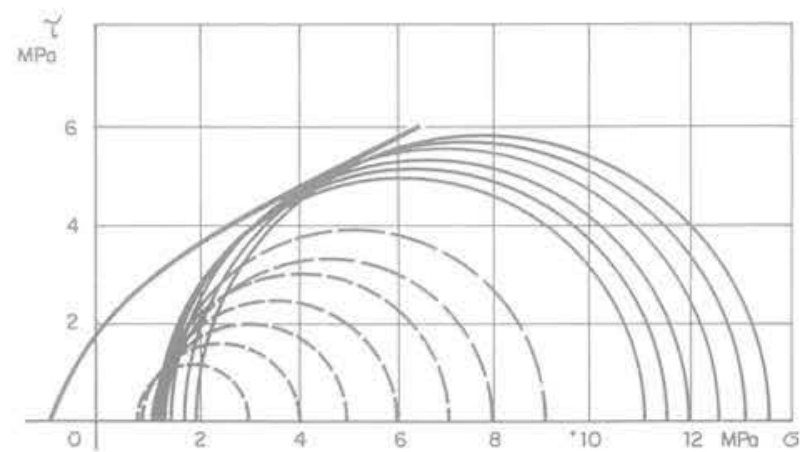

Fig. 1b. - Courbe enveloppe de Mohr au cours d'essais avec expansion latérale contrôlée.

construits pour un échantillon essayé dans l'installation avec la restriction contrôlée de la dilatation latérale.

En arrivant sur la surface enveloppe des cercles de Mohr, le tenseur de contraintes commence à glisser suivant la surface de résistance, ce dont témoignent tous les cercles de Mohr suivants qui sont tangents à l'enveloppe.

Ce glissement du tenseur des contraintes suivant la surface limite de résistance est plus évident sur la courbe de résistance construite en coordonnée $\left(\sigma_{1}-\right.$ $\left.\sigma_{3}\right)$ et $\left(\sigma_{1}+\sigma_{3}\right)$ et présentée sur la figure 2 .

La sollicitation de l'échantillon jusqu'à sa rupture a lieu suivant la ligne droite inclinée, dont l'angle d'inclinaison est déterminé par la rigidité du massif encaissant: plus la rigidité du massif est grande (plus la restriction de la dilatation latérale de l'échantillon est importante), plus l'angle d'inclinaison de cette droite sur l'axe des abscisses est petite. Avec l'absence de restriction latérale (essai de compression simple) la droite de charge a une inclinaison de $45^{\circ}$ et joint l'origine des coordonnées au point correspondant à la résistance à la compression simple $\left(\mathrm{R}_{\mathrm{c}}\right)$.

En arrivant à la «surface de rêsistance», le tenseur des contraintes commence à glisser suivant cette dernière. Ce procédé permet d'obtenir au cours des essais d'un seul échantillon non seulement un point de la surface de rupture, quỉ est généralement le résultat des essais classiques avec les appareils triaxiaux, mais toute une zone de cette surface. 
$\sigma_{1}-\sigma_{3}$

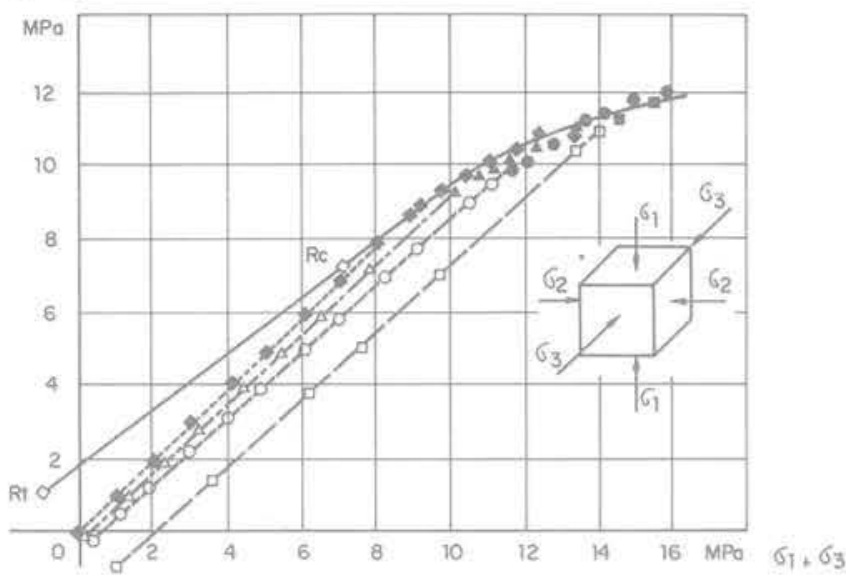

Fig. 2. - Courbe limite pour des échantillons intacts de gypse.

Si l'échantillon est déchargé et ensuite de nouveau rechargé, il atteindra la surface de résistance au point même où le déchargement a commencé. Autrement dit, dans les conditions de restriction de la dilatation latérale, l'échantillon devient plus résistant après la rupture grâce à la naissance des contraintes latérales qui ne disparaissent pas complètement lors du déchargement. On peut donc dire que pendant la rupture, l'échantillon se bloque lui-même dans le massif, en créant une contrainte latérale supplémentaire due à l'augmentation de volume provoquée par la rupture. Si cette contrainte latérale supplémentaire est enlevée, l'échantillon écrasé se comporte de nouveau pendant son rechargement comme un échantillon intact, non remanié.

Les résultats des essais itératifs des échantillons déjà écrasés sont présentés sur la figure 3 .

II en résulte la conclusion très importante mais paradoxale que la résistance du matériau rocheux dans les conditions d'une restriction même insignifiante de la dilatation latérale ne diminue pratiquement pas après la rupture. Vous pouvez voir les faces d'un échantillon cubique après la rupture et avant la deuxième épreuve sur la figure 4.

L'utilisation des coordonnées $\left(\sigma_{1}-\sigma_{3}\right)$ et $\left(\sigma_{1}+\sigma_{3}\right)$ est admissible seulement dans le cas oú deux des trois contraintes principales sont égales $\left(\sigma_{2}=\sigma_{3}\right)$. En général, il est indispensable d'utiliser les trois contraintes principales dans la représentation et l'analyse des résultats.

La forme la plus utile de la représentation des résultats d'essais des matériaux rocheux dans l'état de contrainte triaxial est l'utilisation en tant que coordonnées, du premier invariant du tenseur des contraintes:

$$
\sigma^{1}=\sigma_{1}+\sigma_{2}+\sigma_{3}
$$

qui est le triple de la contrainte normale movenne et de la racine carrée du deuxième invariant du déviateur du tenseur des contraintes:

$\sqrt{\sigma_{\mathrm{d}}^{\mathrm{n}}}=\sqrt{\left(\sigma_{1}-\sigma_{2}\right)^{2}+\left(\sigma_{2}-\sigma_{3}\right)^{2}+\left(\sigma_{3}-\sigma_{1}\right)^{2}}(3)$ représentant trois fois la contrainte tangentielle octaedrique.

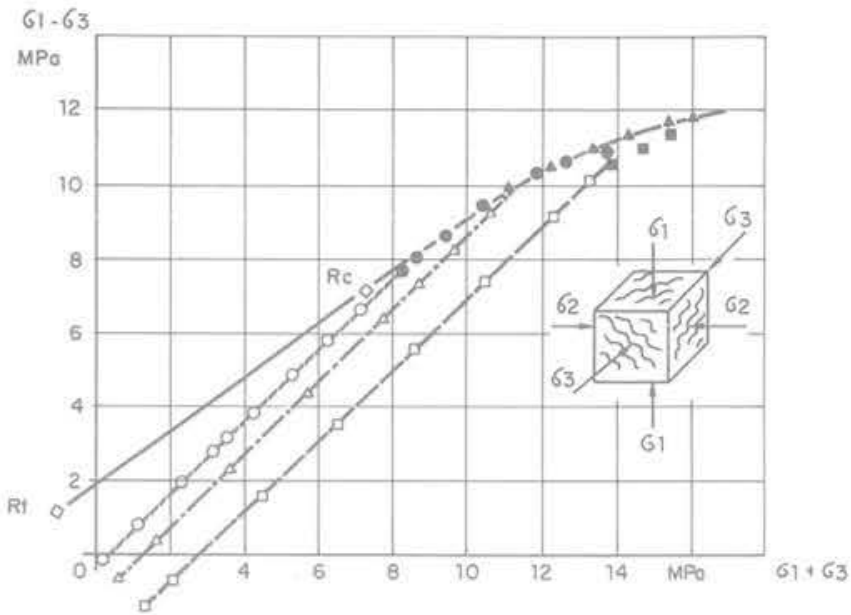

Fig. 3. - Courbe limite pour des échantillons préalablement écrasés.
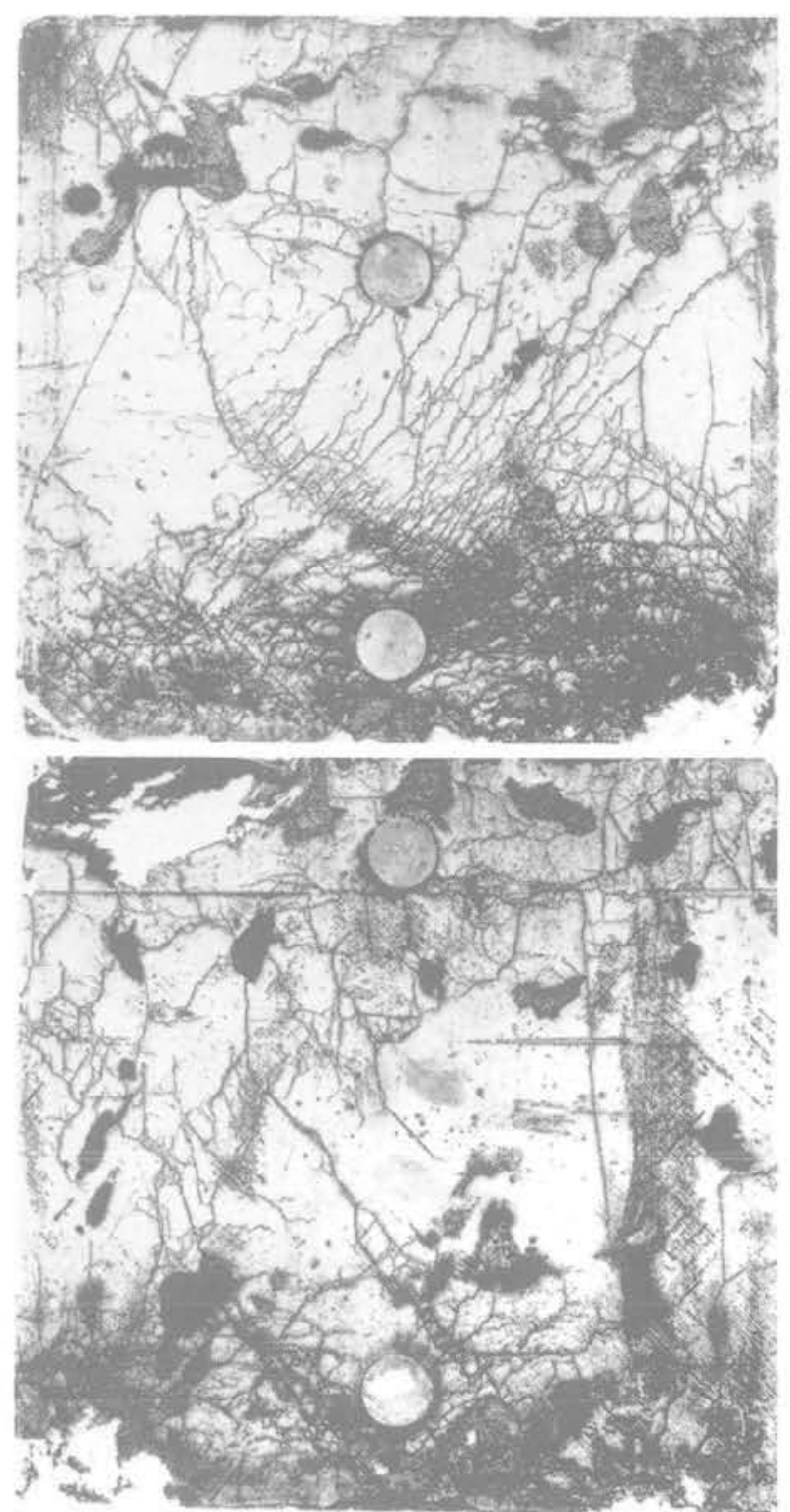

Fig. 4. - Les faces d'un échantillon cubique après la rupture et avant la deuxième épreuve. 
Pour la représentation des résultats sous une forme adimensionnelle permettant de comparer les données obtenues par divers auteurs avec des roches différentes, nous proposons d'utiliser les coordonnées suivantes:

$$
\begin{aligned}
& \sigma_{*}=\frac{\sigma^{\mathrm{I}}}{\mathrm{R}_{\mathrm{c}}} \\
& \tau_{*}=\sqrt{\frac{\sigma_{\mathrm{d}}^{\mathrm{II}}}{2 \mathrm{R}_{\mathrm{c}}^{2}}}
\end{aligned}
$$

où $\mathrm{R}_{\mathrm{c}}$ est la résistance du matériau à la compression simple.

Le diagramme $\tau_{*}=f(\sigma \cdot)$ de la figure 5 représente nos résultats et les résultats obtenus par d'autres secteurs. Tous ces résultats témoignent de la relation étroite entre les coordonnées $\tau$ * et $\sigma$ * où le point $(1,1)$ correspond à la résistance à la compression simple, c'est-à-dire: $\sigma_{1}=R_{c} ; \sigma_{2}=\sigma_{3}=0$.

\section{ETUDE DE L'ÉMISSION ACOUSTIQUE PENDANT LE CHARGEMENT TRIAXIAL DES ÉCHANTILLONS}

L'utilisation de l'émission acoustique permet d'étudier les processus de la formation des fissures ayant lieu dans les matériaux pendant leur chargement.

L'émission acoustique est provoquée par les processus d'autogénération et de propagation dans le matériau des ondes élastiques des contraintes. En ce qui concerne les essais de matériaux rocheux, on peut distinguer deux niveaux de l'émission acoustique en fonction de la nature des sources:

- microniveau lié au maclage, glissement intergranulaire ou à l'intérieur des grains minéraux et aux dislocations entre ces derniers:

- macroniveau lié à la fissuration et à la rupture de volumes de matériau plus importants ou au déplacement réciproque de ses parties structurales.

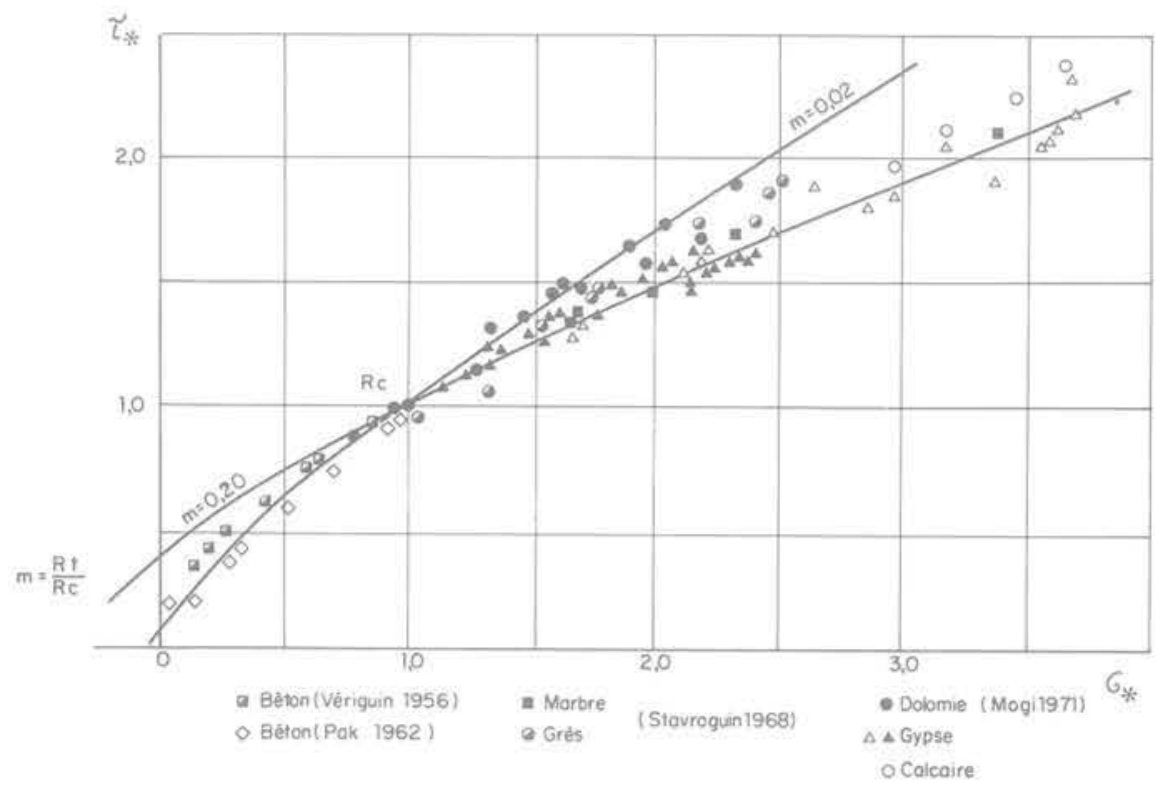

Fig. 5. - Courbe limite en coordonnée $\tau_{*}$, $\sigma$. d'après les résultats expérimentaux de différents auteurs.

L'existence d'une telle relation valable pour les différents types de roches permet de considérer la courbe limite obtenue $\tau_{*}=\mathrm{f}\left(\sigma_{*}\right)$ comme une expression gênéralisée de la surface de résistance.

Pour la description analytique de cette surface de résistance on peut utiliser la relation:

$$
\begin{aligned}
& \text { o. }+\mathrm{m}=(1+\mathrm{m})\left(\frac{\tau \cdot-\mathrm{m}}{1-\mathrm{m}}\right)^{1,3} \\
& \text { où : } \quad \mathrm{m}=\frac{\mathrm{R}_{\mathrm{t}}}{\mathrm{R}_{\mathrm{c}}}
\end{aligned}
$$

$R_{\text {t }}$ résistance du matériau à traction,

$\mathrm{R}_{\mathrm{c}}$ résistance à la compression simple.
Nous avons utilisé une installation qui a permis d'enregistrer l'amplitude des impulsions, leur intensité (nombre d'impulsions de l'émission acoustique pendant une seconde), la puissance des impulsions et leur quantité totale. Les mesures des paramètres de l'émission acoustique ont été effectuées tant au cours des essais à la compression simple des échantillons que pendant leur chargement triaxial.

Toutefois indépendamment du schéma de chargement, on peut déceler les trois stades suivants de la propagation des signaux de l'émission acoustique:

- premier stade: accroissement successif de tous les paramètres de l'émission acoustique; 
- deuxième stade: stabilisation de tous les paramètres;

- troisième stade: augmentation brusque et rapide de tous les paramètres de l'émission acoustique, qui témoigne du commencement de la macrorupture du matériau, autrement dit de "la limite de résistance du matériau s.

La figure 6 représente très nettement tous les trois stades sur un échantillon de gypse essayé en chargement uniaxial. Les essais montrent que, d'habitude, juste avant le commencement du troisième stade, c'est-à-dire avant que la limite de résistance du matériau soit atteinte, sur le diagramme de l'émission acoustique surgit un «signal aigu» annonçant la rupture.

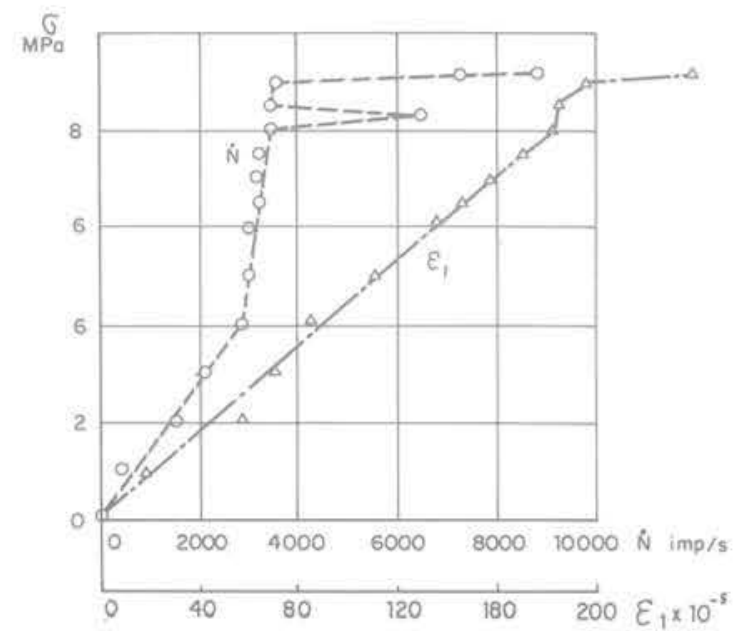

Fig. 6. - Variation de l'intensité de l'émission acoustique pendant un essai de compression simple.

Il faut souligner que les signaux de l'émission acoustique surviennent presque immédiatement après l'application de la charge à l'échantillon. Cela témoigne du fait que la formation et le développement des fissures commencent immódiatement avec le début du chargement de l'échantillon, en passant, au fur et à mesure de l'accroissement de la charge, du microniveau au macroniveau.

\section{ETUDE DES DÉFORMATIONS D'UN ÉCHANTILLON PENDANT SON CHARGEMENT TRIAXIAL}

Les déformations des échantillons sous charge ont été mesurées à l'aide de capteurs installés directement sur les faces de ces derniers.

Le dépouillement et l'analyse des résultats ont été effectués sous forme d'une relation entre la racine carrée du deuxième invariant du déviateur des déformations:

$\sqrt{\varepsilon_{\mathrm{d}}^{\pi}}=\sqrt{\left(\varepsilon_{1}-\varepsilon_{2}\right)^{2}+\left(\varepsilon_{2}-\varepsilon_{3}\right)^{2}+\left(\varepsilon_{3}-\varepsilon_{1}\right)^{2}}$ et la racine carrée du deuxième invariant du déviateur des contraintes:

$\sqrt{\sigma_{d}^{\pi}}=\sqrt{\left(\sigma_{1}-\sigma_{2}\right)^{2}+\left(\sigma_{2}-\sigma_{3}\right)^{2}+\left(\sigma_{3}-\sigma_{1}\right)^{2}}(9)$ où $\sigma_{1}, \sigma_{2}$ et $\sigma_{3}$ sont les contraintes principales sur les faces de l'échantillon cubique essayé et $\varepsilon_{1}$, $\varepsilon_{2}$ et $\varepsilon_{3}$ sont les déformations relatives de l'échantillon dans les directions correspondantes.

Sur la figure 7 est représenté le diagramme de cette relation pour les échantillons «intacts» et écrasés pendant leurs essais itératifs. II est assez facile de se persuader que dans les coordonnées indiquées les résultats d'essais sont étroitements liés entre eux, et la relation entre les composantes du tenseur des déformations et celles du tenseur des contraintes est linéaire jusqu'à la rupture et l'angle d'inclinaison de cette relation à l'axe des déformations caractérise le module de déformation ou le module de cisaillement du matériau essayé:

$\frac{\sqrt{\sigma_{d}^{\pi}}}{\sqrt{\varepsilon_{d}^{\pi}}}=\frac{E}{1+v}=2 G$

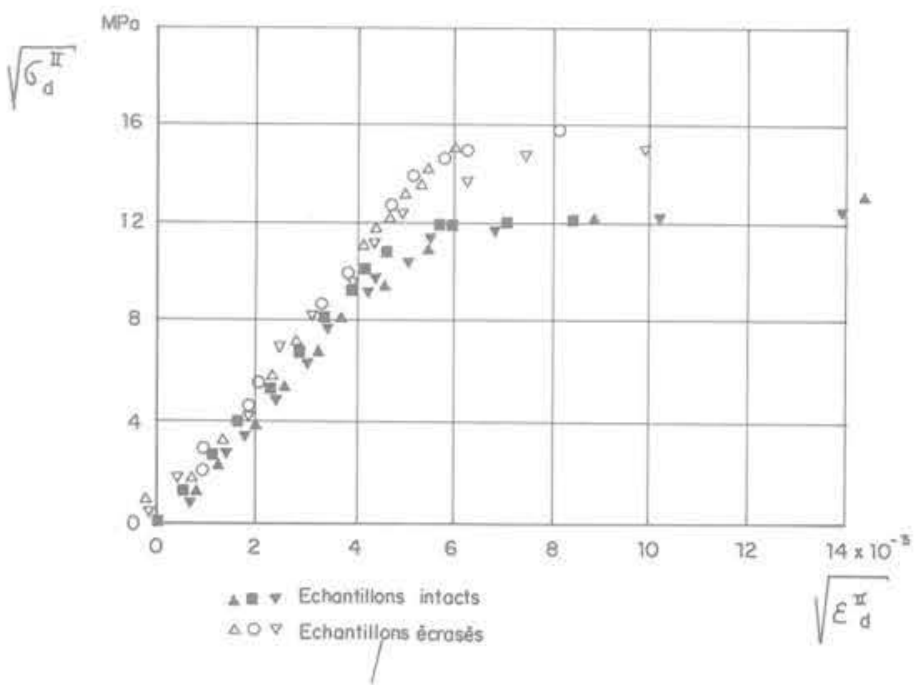

Fig. 7. - Diagramme de déformation.

Pendant la rupture, a lieu une brusque diminution du module de déformation (ou une brusque augmentation du coefficient de dilatation (latérale) et, après l'enlèvement de la charge, au cours de son rechargement l'échantillon travaille, après une charge déterminée, de nouveau d'une manière élastique avec un module de déformation un peu plus grand.

La figure 8 représente le diagramme de chargement et de déchargement d'un échantillon de gypse, permettant de voir son «renforcement » ou écrouissage après la rupture et sa consolidation assurant l'accroissement du module de cisaillement et, donc, du module de déformation. 


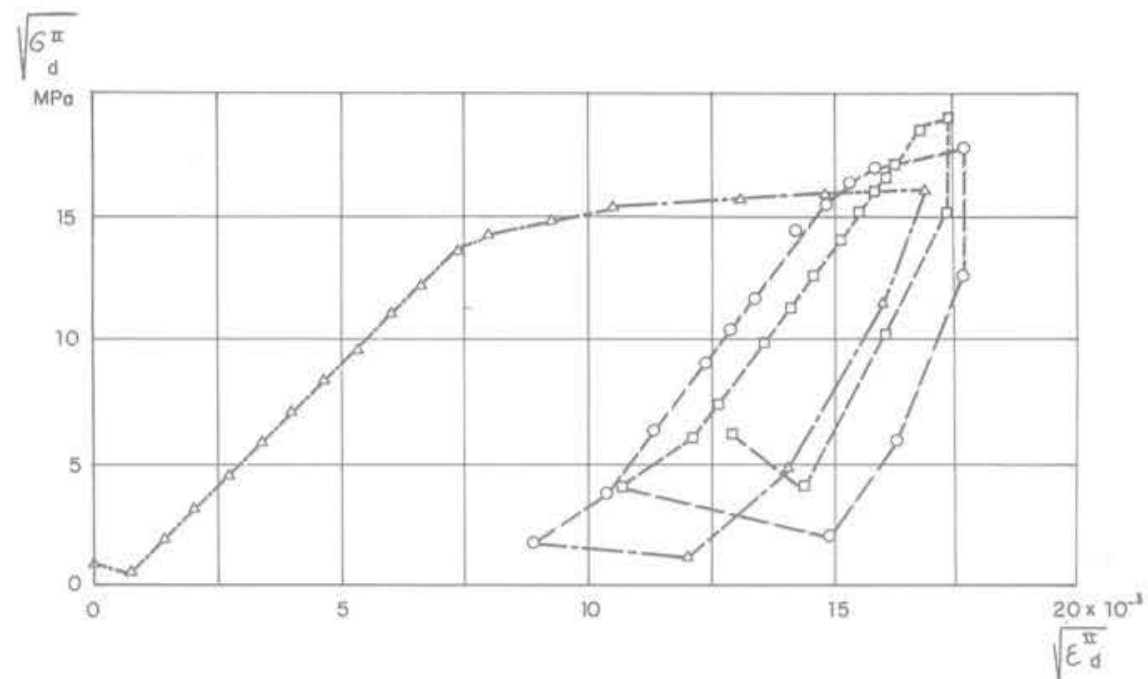

Fig. 8. - Diagramme de déformation (chargementdéchargement) d'un échantillon de calcaire en coordonnées $\sqrt{\sigma_{d}^{\pi \prime}} \sqrt{\varepsilon_{d}^{\| \prime}}$. 